\title{
CONTIGO NO BITXO, INICIATIVA DE ACERCAMIENTO DEL EQUIPO DE CONSERVACIÓN A COLECTIVOS ARTÍSTICOS
}

\author{
Colectivo Contigo no Bitxo: \\ Katrin Alberdi Egués \\ Jose Luis Larrañaga Odriozola \\ Haizea Salazar Basañez \\ Universidad del País Vasco/Euskal Herriko Unibertsitatea. Dpto. Pintura
}

\section{Resumen}

¿Cuáles son las herramientas de la conservación-restauración? Seguramente lo primero que nos venga a la cabeza sea la limpieza de obras, su arreglo, etc. pero desde hace tiempo la entrevista ha comenzado a tomar importancia. La entrevista es un método de documentación que permite conservar la postura de las/os artistas. No obstante, la manera de abordarla tiene distintos enfoques y todavía, debido a la brecha entre la conservación-restauración y la creación, este acercamiento puede entenderse como un ataque en vez de como una aproximación. Es imprescindible conocer las obras y sus complejidades. Por ello, las jornadas/congresos dedicadas a la problemática del arte contemporáneo son cada vez más habituales si bien es cierto que la visión que se expone pertenece al área de conservación-restauración. Sin embargo, ¿y si continuamos evolucionando este concepto y dejamos que sean los/as artistas quienes nos cuenten sus casuísticas? Al fin y al cabo, no hay mejor información que la de primera mano.

\section{Palabras clave: ARTE CONTEMPORÁNEO; CONSERVACIÓN; ARTISTAS; DIÁLO- GOS; JORNADAS}

\section{CONTIGO NO BITXO INITIATIVE, AN APPROACHING STRATEGY FROM CONSERVATORS TO ART COLLECTIVES}

\begin{abstract}
Which are conservation-restoration's tools? Surely, the first thing that comes to mind is the cleaning of artworks, their care, etc. However, while ago, the interview has begun to take on importance. The interview is a documentation method to preserve the artists' opinion. Nevertheless, its tackle has different ways and due to the gap between conservation-restoration and creation, it still can be understood as an attack rather than as an approach. It is essential to understand artworks and their complexities. For this reason, the conferences dedicated to the problem of contemporary art are increasingly common, although it is true that the vision that is exposed focuses in to conservation-restoration. However, what if we continue developing this concept, letting the artists tell us about their own casuistry? After all, there is no better information than first-hand information.

Keywords: CONTEMPORARY ART; CONSERVATION; ARTISTS; DIALOGUES; SYMPOSIUM

Alberdi Egués, Katrin, Jose Luis Larrañaga Odriozola \& Haizea Salazar Basañez. 2021. "Contigo no Bitxo, Iniciativa de acercamiento del equipo de conservación a colectivos artísticos". AusArt 9 (2): 19-30. D0I: 10.1387/ ausart.23107
\end{abstract}

\section{AUSART}




\section{INTRODUCCIÓN}

La preocupación por la conservación del arte contemporáneo es un tema de interés para los/as profesionales del arte desde hace años. Sin embargo, generalmente, se aborda y se discute una vez aparecen los deterioros en las obras de arte.

El siglo $X X$ trajo grandes cambios que dieron como resultado nuevas posturas frente al arte, y algunas de estas se manifestaron como rechazo frente a la restauración, ideología que actualmente siguen compartiendo algunas/os artistas, y que desde el punto de vista de la conservación-restauración no tendría que ser un problema, siempre y cuando fuese una decisión basada en la reflexión. Sin embargo, se ha observado que, en ocasiones, esta elección no se sustenta en una filosofía o resistencia, sino en el desconocimiento; una falta de conciencia y de diálogo entre ambas disciplinas que nace, habitualmente, desde la formación.

Según Ana Iribas Rudín (2015) el arte contemporáneo se construye sobre un maridaje constituido por la figura del/la artista y su libertad creadora, la diversidad de los materiales empleados, y el mercado del arte. La actitud desde la que el/la artista trabaja constituye el punto de partida y el condicionante fundamental de la creación. A partir de ahí hace falta considerar múltiples factores, como la sociedad y la cultura en su conjunto, que la determinan y la convierten, desde un punto de vista conceptual, en productos culturales evaluados de acuerdo con la demanda de lo nuevo.

Así, las relaciones establecidas entre los sujetos involucrados en la producción, deleite y preservación del arte contemporáneo se presentan como algo complejo y novedoso. Dentro de estas relaciones, el equipo de conservación-restauración será la que protagonice las actuaciones frente a las cuestiones relacionadas con el deterioro involuntario que se escapan al control del/la artista. La preservación de la idea será el eje central que deberá defender ante la materialidad de la obra. Para entender el concepto de la obra a preservar resulta imprescindible obtener la máxima información sobre su significado, y por ello, resulta fundamental establecer un diálogo directo con las/os artistas con el fin de comprender todos los planos que conforman sus propuestas artísticas. En este contexto, las entrevistas se han convertido en las últimas décadas en una herramienta esencial para el equipo de conservación-restauración. Basándose en el interés y la comprensión, no solo del trabajo artístico sino como una sinergia de conocimiento, cada vez es más habitual la cola- 
boración multidisciplinar para el diseño conjunto de la intervención (De Sousa-Júnior \& Llamas 2019).

Dado que la colaboración artista-conservador/a es relativamente reciente, todavía es común que algunos/as artistas perciban estas entrevistas como un interrogatorio. Por ello, es necesario desarrollar nuevos escenarios y vías de acercamiento que no se limiten únicamente al equipo de conservación y artistas, sino que contemplen otros colectivos como galeristas, curators, o equipos de montaje.

\section{RELACIÓN DEL COLECTIVO ARTÍSTICO/ EQUIPO DE CONSERVACIÓN-RESTAURACIÓN}

Los/as profesionales de la conservación -restauración detectamos por parte de la comunidad artística cierto recelo que puede atribuirse al desconocimiento y/o a una percepción de la profesión ya obsoleta que se identifica con modos de hacer y tratamientos basados en obra tradicional. La continua experimentación del arte contemporáneo ha 'obligado' al equipo de conservación-restauración, entre otras cosas, a estudiar nuevos materiales y establecer nuevos criterios. Resulta preciso conocer exhaustivamente la intencionalidad de la obra, y para ello se considera esencial la comunicación directa con el/la artista (Llamas 2014).

El respeto hacia la obra consiste en entender de antemano qué factores son los importantes y, por lo tanto, no susceptibles de ser alterados. El conocimiento y entendimiento de las obras a nivel conceptual es uno de los pasos previos de cualquier intervención, o no intervención, en arte contemporáneo, de ahí que la colaboración con el/la artista sea fundamental. Tanto a nivel matérico como plástico, el equipo de conservación-restauración se enfrenta a un sinfín de estrategias artísticas y procedimientos experimentales, y se vuelve imprescindible comprender el proceso creativo y la intención, especificando hasta qué punto son aceptables los signos del envejecimiento en una obra de arte, qué se considera alteración, o si es preferible la refabricación o sustitución frente a la restauración.

La comunicación y cooperación durante el proceso creativo puede ser beneficiosa para ambas partes. Para el/la artista en términos de producción y 
ampliación de posibilidades (en cuanto a conocimientos de materiales y técnicas se refiere) y para el equipo de conservación-restauración para conocer en profundidad los diversos planos que conforman la obra obteniendo, además, una valiosa información para su futura conservación. (Llamas 2010; Fuentes et al. 2017).

No hay un momento específico, pero la percepción del/la artista hacia los/ as profesionales de la conservación-restauración se modifica con el tiempo. Habitualmente este cambio suele darse cuando se trata de artistas con una trayectoria consolidada, con experiencia artística en montaje de exposiciones, en almacenaje de obra y manipulación de su producción, y principalmente cuando las cuestiones de conservación de su obra se vuelven un asunto a contemplar dentro de su relación con la productividad artística en el mercado. De esta manera, parece que el rechazo inicial por parte del colectivo artístico hacia el equipo de conservación y restauración se disipa y se trasforma en comunicación y cooperación.

\section{LA ENTREVISTA COMO MÉTODO}

Como se ha comentado, actualmente, la entrevista ha adquirido una importancia destacada en el ámbito de la conservación-restauración y preservación del arte contemporáneo llegando a considerarse como un modo de conservación preventiva (Fresno 2017).

A mediados de la década de los sesenta del siglo $X X$ referentes del ámbito de la conservación-restauración como Heinz Althöfer y Hiltrud Schinzel comenzaron a recabar información sobre obras de arte contemporáneo. Valiéndose de formularios obtenían información referente a los/as artistas y su modo de trabajar como por ejemplo el tipo de materiales que utilizaban en la ejecución de sus obras (Althöfer 2003).

A partir de los años 80, Joyce Hiel Stoner, del centro Ralph Mayer de documentación de técnicas artísticas de la Universidad de Delaware (Estados Unidos), realizó una serie de fichas técnicas específicas para la conservación. En el Instituto de restauración del Instituto de Arte de Dresde (Alemania) también se elaboraron cuestionarios dirigidos a artistas, donde se les preguntaba acerca del envejecimiento de sus obras (Chiantore \& Rava 2005). 
Desde el inicio de la década de los 90 Carol Mancusi-Ungaro, conservadora de la colección Menil de Houston (Estados Unidos), desarrolló una investigación basada en la recopilación de información sobre las obras de arte entrevistando a artistas y sus colaboradores/as con el fin de obtener una mejor comprensión de sus materiales, técnicas de trabajo e intención de conservación de sus obras (INCCA, International Network for the Conservation of Contemporary Art).

Esto marcó el inicio de ADP (Programa de Documentación de Artistas), el primer proyecto en el que las/os artistas fueron invitados/as por una institución de arte a dialogar sobre el futuro de su trabajo. Siguiendo los pasos de Mancusi-Ungaro, en la segunda mitad de los noventa, se gestó otra iniciativa, esta vez en los Países Bajos. Dentro del innovador proyecto de investigación Arte moderno: ¿A quién le importa?, se discutió extensamente la participación del/ la artista en la preservación del arte contemporáneo. Tanto el proyecto como el simposio internacional resultante tuvieron un gran impacto en el campo del arte moderno y contemporáneo. Se convirtió en inspiración para otras iniciativas de investigación, como Entrevistas de artistas y Archivos de artistas, realizadas en los Países Bajos entre 1998 y 2005 por SBMK (Stichting Behoud Moderne Kunst).

En 1999 se estableció INCCA (International Network for the Conservation of Contemporary Art), una plataforma diseñada para recopilar, compartir y preservar conocimientos valiosos para la conservación del arte contemporáneo. El objetivo de esta iniciativa era doble: en primer lugar, desarrollar pautas para entrevistar a artistas y, en segundo lugar, construir un espacio virtual que permitiese el intercambio de información y conocimientos entre profesionales sobre temas contemporáneos (Wielocha 2018).

Se popularizaron iniciativas donde se compartía la recopilación de entrevistas a artistas como punto de información a diferentes niveles. Es el caso de SFMOMA (San Francisco Museum of Modern Art)(sfomoma.org) que reúne en su página web el proceso creativo y las historias de muchos/as artistas que conforman su colección, aunque su perspectiva no es la de la conservación. Lo mismo ocurre con el Museo de Arte Contemporáneo S.M.A.K. de Gante (Bélgica), que recopila algunas entrevistas a artistas sobre su proceso creativo en la plataforma ARTIST TALK (smak.be).

Siguiendo este hilo, The Getty Conservation Institute, con su programa ART IN L.A. genera diálogos de artistas. La serie de videos Artist Dialogues involucra 
al colectivo de artistas en conversaciones que exploran su arte, materiales, procesos de fabricación y métodos de trabajo, así como sus pensamientos sobre la conservación, haciendo que la información sea accesible al colectivo de conservadores/as y otros colectivos como curators, coleccionistas, educadores/as o historiadores/as del arte y generando foros públicos sobre nuevas formas de colaboración y documentación ${ }^{1}$.

La entrevista, como fuente de información directa y primaria, es una herramienta que tiene una eficacia indiscutible, pero, en ocasiones, no es suficiente para afrontar los desafíos inherentes a la conservación del arte contemporáneo, sin obviar, que las ideas plasmadas pueden ser susceptibles de cambio.

\section{LOS CONGRESOS Y JORNADAS SOBRE LA CONSERVACIÓN DE ARTE CONTEMPORÁNEO}

En todo el mundo se realizan actualmente congresos y jornadas en torno a la temática de la conservación-restauración del arte contemporáneo que pretenden crear enlaces y compartir conocimientos sobre las nuevas problemáticas en este campo. Se reflexiona y estudia desde diferentes prismas la creación artística, los materiales, la importancia de la documentación... y parece que todas tienen una característica común: la presencia de los/as artistas suele ser nula o residual. Esto supone que el protagonismo recae en otras disciplinas que trabajan con el arte, especialmente en los/as profesionales de la conservación-restauración.

Como referentes de congresos de conservación-restauración internacionales son destacables los organizados por el NACCA (New Approaches in the Conservationn of Contemporary Art). En este congreso no hay presencia de artistas, solo conservadores/as y otros perfiles relacionados con el arte como representantes de instituciones, investigadores/as, profesorado de universidades, comisariado, etc. Lo mismo ocurre con las jornadas organizadas a través de Contemporary Conservation ${ }^{\text {Itd }}$, desde un estudio privado de Nueva York donde se tratan los temas relacionados con la conservación de arte contemporáneo, pero con una presencia anecdótica de artistas, siendo protagonistas otras disciplinas relacionadas con el arte.

Desde el programa de grado Conservación y Restauración de la UVA, en colaboración con la Fundación para la Conservación del Arte Conteporáneo 
(SBMK) y la Agencia de Patrimonio Cultural de los Países Bajos (RCE), se han organizado talleres relacionados con la conservación del arte contemporáneo con un enfoque para el colectivo de comisariado, equipo de conservación-restauración e historiadores/as del arte. En este caso tampoco se contempla la presencia de artistas como colaboradores directos (University of Amsterdam).

Desde el ICON, The Institute of Conservation (UK) se lanzan conferencias online sobre la conservación de arte contemporáneo con la participación del sector artístico dejando fuera a los/as $\mathrm{s}$ artistas ye repite la dinámica en la Jornada Nacional sobre Conservación de Arte Contemporáneo del Museo de Bellas Artes de Buenos Aires, Argentina.

En el estado español se organizan de manera anual con una trayectoria de más de 20 años las jornadas de Conservación de Arte Contemporáneo en el Museo Nacional de Arte Contemporáneo del Reina Sofía de Madrid, unas jornadas que tienen como objetivo compartir experiencias e investigaciones, debatir y sugerir nuevas vías de estudio sobre este campo, así como reflexionar acerca de la gestión institucional de la conservación y la práctica profesional del restaurador/a. En este caso también, la participación directa de artistas suele ser escasa, dando preferencia a discursos desde otros sectores profesionales.

En este panorama, se puede apreciar que por un lado se considera imprescindible la experiencia directa del/la artista, como se ha demostrado desde el desarrollo de las entrevistas y su registro por parte de los/as profesionales de la conservación; y por otro, la importancia de las jornadas donde se muestran las investigaciones realizadas en el campo de la conservación-restauración del arte contemporáneo de la mano de diferentes sectores que trabajan en el área para poder expandir los conocimientos y generar reflexiones acerca de las nuevas problemáticas y las soluciones planteadas. Pero, ¿por qué no unir los dos componentes y generar una nueva propuesta híbrida?

\section{DE LO INTERNACIONAL A LO LOCAL}

Es cierto que si echamos una mirada global al panorama artístico mundial podemos sentir cierto vértigo al comenzar a plantear iniciativas relacionadas con nuevas propuestas indiferentemente del tipo que sean. Por ello es importante otear la realidad más cercana para establecer un punto de partida que 
permita desarrollar un proyecto inicial al cual se podrá dotar de un desarrollo más complejo, o más amplio, según se vayan definiendo las necesidades.

Se ha establecido la realización de las entrevistas a los/as artistas como clave en las intervenciones de conservación-restauración en el arte contemporáneo. Pero la proporción entre las/os profesionales del arte que se encuentran en activo y las entrevistas realizadas (y accesibles) son mínimas. Las grandes plataformas como INCCA o VOCA recogen de manera pública entrevistas con artistas internacionales (algunos/as ya fallecidos/as). A partir de ahí, se han desarrollado diferentes iniciativas por parte de museos y universidades para registrar entrevistas de artistas nacionales y locales. Estas en cambio no tienen plataformas online para compartir los contendidos. No existen redes globales de bases de datos unificadores, esto sería ciertamente muy difícil de abarcar.

En la Facultad de Bellas Artes de la Universidad del País Vasco (EHU/UPV) se llevan a cabo diferentes iniciativas relacionadas con conversaciones con artistas, como el Proyecto Archivo/Artxibo Proiektua, para conocer su producción, los materiales, técnicas, así como su concepción artística y conceptualidad de la obra.

Además, en el máster CYXAC (Conservación y exhibición de Arte Contemporáneo), de la misma facultad, se llevan a cabo entrevistas a los/as artistas vascos/as que conforman la colección de la UPV/EHU con un enfoque específico en conservación.

Las guías referentes de las entrevistas de artistas creadas por el INCCA, o los diálogos registrados por VOCA, se centran en la relación artista-entrevistador/a siendo siempre un perfil de conservador/a quien previamente ha preparado las preguntas específicas. Nunca se plantea una conversación entre artistas, dejando que sean protagonistas únicos/as, en la que el equipo de conservación-restauración, pese a generar un guion de preguntas de interés conservativo, quede relegado a un segundo plano. Un espacio donde surjan diálogos inesperados, enriquecedores y sinceros entre las diferentes combinaciones de parejas artísticas, mientras se perfilan nuevas sinergias, se acercan posturas y se acortan distancias. 


\section{OBJETIVOS, METODOLOGÍA Y RESULTADOS DE LA INICIATIVA 'CONTIGO NO BITXO'}

'Contigo no Bitxo' es un experimento a distintos niveles que surgió en 2018 desde el Grado en Conservacion y Restauración de Bienes Culturales de la UPV/EHU tras la reflexión del estado actual de la relación entre el colectivo de conservación-restauración, el colectivo artístico, el arte contemporáneo y sus necesidades. Esta iniciativa indaga, no solo una manera alternativa de presentar las problemáticas reales del arte contemporáneo desde distintos prismas, sino que pretende, además, crear un espacio de diálogo y discusión libre en el que los propios colectivos narren sus experiencias y valoren, desde su perspectiva, la necesidad, o no, del equipo de conservación en sus realidades, visibilizando ante el propio colectivo artístico una reflexión sobre la conservación en sus creaciones. Esta propuesta no tiene como finalidad principal ahondar en detalle los procesos de elaboración de las obras, ni los materiales ni las técnicas empleadas como sucedía con las entrevistas a artistas que hemos visto hasta ahora realizadas desde el colectivo de la conservación-restauración.

Aun siendo materias que se imparten en un mismo centro, se considera que el abismo que existe entre la creación y la conservación-restauración es uno de los mayores problemas. Las diversas experiencias lectivas durante los años de educación revelan que los/as futuros/as artistas carecen de un mínimo de formación en lo que a la conservación se refiere, siendo, en ocasiones, los desperfectos que sufren sus obras consecuencia de una falta de conocimiento.

Para evitar que este deterioro sea producto del desconocimiento y no de una intención, 'Contigo no Bitxo' propone acercar estas dos disciplinas, conservación-restauración y creación, de manera abierta a todos los agentes que participan, activa o pasivamente, en el plano artístico. Ya que las jornadas están enfocadas principalmente para el alumnado y se organizan anualmente en la Fundación BilbaoArte de manera gratuita. El enclave de las jornadas también fue un tema sobre el que se reflexionó en profundidad y finalmente la decisión fue la Fundación BilbaoArte, por tratarse de un referente a nivel local y nacional, como centro de producción artística.

Tras las ediciones de las jornadas 'Contigo no Bitxo' de 2019 y 2020 (imagen 1 y 2), se puede afirmar que el cambio de formato propuesto para las ponencias ha generado una sinergia que ha dado como resultado un discurso fluido de los/as participantes, así como el desarrollo de temas que albergaban mucho interés y reflexión más allá de la discernida conversación. Las distintas res- 
puestas, desde diversas ópticas, a las mismas preguntas han sido el germen para que este proyecto colectivo haya comenzado a acortar las distancias de dos mundos, tan unidos y a la vez tan separados, como el arte y la conservación. La creación de este entorno ha ayudado no solo a acercar posturas y personas, sino que ha servido como inspiración para futuros temas de investigación al poder acceder de primera mano a las inquietudes que el arte se plantea.

Las grabaciones de video han facilitado su divulgación y proporcionarán a los/ as futuros conservadores/as y académicos/as un contexto que de otro modo se habría perdido en una mera transcripción.

\section{REFLEXIONES FINALES. CONCLUSIONES}

Si bien es cierto que la entrevista al artista es una herramienta muy útil para la conservación de arte contemporáneo, también es cierto que es necesario adaptarla para construir relaciones de confianza entre artistas y conservadores/as, así como para no limitarla a un mero interrogatorio sobre la técnica o la preservación.

Por otro lado, aunque los congresos y jornadas sobre la conservación de arte contemporáneo son muy necesarios para esta profesión, lo cierto es que en contadas ocasiones ayudan a crear relaciones entre artistas y conservadores/ as.

"Contigo no Bitxo" apuesta por las conversaciones frente a las entrevistas haciendo de esta manera que los/as artistas y otros/as profesionales del sector sean los/as protagonistas al aportar su conocimiento a través de sus experiencias y vivencias personales. Así mismo, aunque sabemos que la opinión es susceptible al cambio, consideramos importante el registro de estas conversaciones con el fin de facilitar su consulta y accesibilidad al conocimiento abierto.

Para concluir, consideramos que el proyecto 'Contigo no Bitxo' no solo ha servido como germen para nuevas colaboraciones artísticas e interdisciplinares, sino que la realización de esta tercera edición, junto con la acogida del público en todas sus ediciones, supone su consolidación en el panorama artístico y de conservación del País Vasco. Un escenario donde hemos visto reflejados 
intereses que nos han sorprendido gratamente además de los problemas que preocupan a artistas tan dispares como Itziar Okariz, Javier Pérez o Esther Ferrer entre otros/as. Una muestra de nuestra capacidad de adaptación y reinvención a los tiempos, y a las necesidades, que pueda contribuir a mejorar las relaciones interdisciplinares y competencias futuras.

\section{Referencias bibliográficas}

Althofer, Heinz. 2003. Restauración de la pintura contemporánea: Tendencias, materiales, técnicas. En colaboración con Hiltrud Schinzel et al.; traducción, Lourdes Rico Martínez, con la colaboración de Karla Kühnen. Madrid: Akal)

Chiantore, Oscar \& Antonio Rava. 2005. Conservare l'arte contemporáea: Problema, metodi, materiali, ricerchi. Milano: Electa

De Sousa Júnior, Mario Anacleto \& Rosario Llamas Pacheco. 2019. "La representación social del sujeto en el díalogo con el artista y el conservador de arte contemporáneo". Arte, Individuo y Sociedad 31(2): 261-76. https://doi.org/10.5209/ARIS.58843

Fresno Guillem, Ruth del. 2017. "La entrevista al artista emergente como modo de conservación preventiva: Estudio aplicado a los proyectos Perspectives Art Inflammation and Me y Perspectives, Art Liver Diseases and Me". Tesis Univ. Politècnica de València

Fuentes Duran, Eva, Mariana Fuentes Duran, Rita Lucía Amor Garcia \& María Pilar Soriano Sancho. 2017. "New insights for creative art processes: Collaboration and experimentation with contemporary artists". Ge-Conservación 11: 257-63. https://doi.org/10.37558/gec. v11i0.502

Iribas Rudín, Ana. 2015. La actitud del artista. Madrid: Clepsidra

Llamas Pacheco, Rosario. 2014. Arte contemporáneo y restauración o Cómo investigar entre lo material, lo esencial y lo simbólico. Madrid: Tecnos

Llamas Pacheco, Rosario. 2010. Conservar y restaurar el arte contemporáneo: Un campo abierto a la investigación. València: Universitat Politècnica de València

Mancusi-Ungaro, Carol, Shelley Sturman \& Erich Gantzert Castrillo. 2000. "Working with artists in order to preserve original intent". In Modern art, who cares? An interdisciplinary research project and an International Symposium on the Conservation of Modern and Contemporary Art, eds., IJsbrand Hummelen \& Dionne Sillé; text ed., Marjan Zijlmans, 391-9. Amsterdam: Netherlands Institute for Cultural Heritage

Scheidemann, Christian. 2016. "Why not ask the artist?". VoCA Journal, Feb. 8. https://journal. voca.network/why-not-ask-the-artist/

VoCA (Voices in Contemporary Art). 2014. "The artist interview workshop: Reflections of a conservator in private practice". May 7. https://voca.network/blog/2014/05/07/reflections-of-a-conservator-in-private-practice/

Wielocha, Aga. 2018. "The artist interview as a platform for negotiating an artwork's possible futures". Art and Documentation 17: 31-45. https://doi.org/10.6084/m9.figshare.7957715.v1 


\section{Recursos web}

Contemporary Conservation Ltd. https://www.contemporaryconservation.com/cc-symposia

ICON (The Institute of Conservation). https://icon.org.uk/about-us/who-we-are

INCCA (International Network for the Conservation of Contemporary Art). Project: Artists Documentation Program (ADP). https://www.incca.org/articles/project-artists-documentation-program-adp

MNCARS (Museo Nacional centro de arte Reina Sofía). https://www.museoreinasofia.es/buscar?bundle=actividad\&f\%5B0\%5D=im_field_obra_clasificaciongener\%3A4243

Museo de Bellas Artes de Argentina. https://www.bellasartes.gob.ar/paginas/encuentro-nacional-sobre-registro-documentacion-y-conservacion-de-arte-contemporaneo/

NACCA (New Approaches in the Conservation of Contemporary Art). http://nacca.eu/

SFOMOMA (San Francisco Museum of Modern Aart). https://www.sfmoma.org/artists-artworks/

SMAK (Gent Stedelijke Museum voor Actuele Kunst). https://smak.be/en/zoeken?q=artist+talk

University of Amsterdam. https://www.uva.nl/en/shared-content/faculteiten/en/faculteit-der-geesteswetenschappen/news/2018/11/acting-in-contemporary-art-conservation.html?cb

Voices in Contemporary Art (VOCA). https://voca.network/

\section{Notas}

1 VOCA Voices in Contemporary Art (VoCA). 2014. "The artist interview workshop: Reflections of a conservator in private practice". May 7. https://voca.network/blog/2014/05/07/reflections-of-a-conservator-in-private-practice/ 